Revue de droit comparé du travail et de la sécurité sociale

3 | 2018

La participation des travailleurs dans la grande entreprise privée et publique

\title{
La cogestion des salariés (Mitbestimmung) en droit allemand
}

Christophe Teichmann et Justin Monsenepwo

\section{OpenEdition}

Journals

Édition électronique

URL : https://journals.openedition.org/rdctss/1882

DOI : $10.4000 /$ rdctss. 1882

ISSN : 2262-9815

Éditeur

Centre de droit comparé du travail et de la sécurité sociale

Édition imprimée

Date de publication : 1 novembre 2018

Pagination : 28-37

ISSN : $2117-4350$

Référence électronique

Christophe Teichmann et Justin Monsenepwo, « La cogestion des salariés (Mitbestimmung) en droit allemand », Revue de droit comparé du travail et de la sécurité sociale [En ligne], 3 | 2018, mis en ligne le 01 novembre 2021, consulté le 13 novembre 2021. URL : http://journals.openedition.org/rdctss/1882 ; DOI : https://doi.org/10.4000/rdctss. 1882

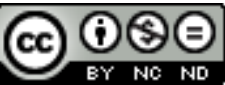

Revue de droit comparé du travail et de la sécurité sociale est mise à disposition selon les termes de la Licence Creative Commons Attribution - Pas d'Utilisation Commerciale - Pas de Modification 4.0 International. 


\title{
LA COGESTION DES SALARIÉS (MitBestimMUnG) EN DROIT ALLEMAND
}

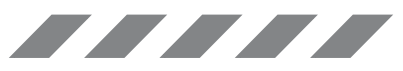

\begin{abstract}
Based on the social principle whereby capital and labour are intended to "co-manage" or "co-determine" (mitbestimmen) the functioning of the company, the co-management of employees (Mitbestimmung) is one of the pillars of the German social partnership. It seeks a balance between the rights of the capital and those of the labour. Therefore, it offsets the considerable weight of the employer within the company by establishing legal representation regimes that allow employees to control and participate in the management of the company through elected or designated representatives. Therefore, without compromising the preponderance of shareholders on the supervisory board, the co-management of employees ensures a permanent social dialogue at the top of the company. This social cohesion constitutes undoubtedly one of the essential elements behind the performance of German companies. This paper not only presents the laws and regulations that govern the co-management of employees but also explores its different legal regimes and its real impact on the functioning of the supervisory board of a company.
\end{abstract}

KEYWORDS : Co-management, Employee Participation, Supervisory Board.

\section{RÉSUMÉ}

Reposant sur une philosophie sociale selon laquelle le capital et le travail ont vocation à « cogérer » ou " co-déterminer » (mitbestimmen) la marche de l'entreprise, la cogestion des salariés (Mitbestimmung) constitue I'un des piliers du partenariat social allemand. Elle recherche un équilibre entre les droits du capital et ceux du travail en compensant le poids considérable de l'employeur au sein de l'entreprise. Pour ce faire, la cogestion instaure des régimes légaux de représentation permettant aux salariés, par l'entremise de représentants élus ou désignés, d'exercer un droit de contrôle et de participer à la gestion ainsi qu'à l'organisation de l'entreprise. Ainsi, tout en ne portant pas atteinte à la prépondérance des actionnaires au sein du conseil de surveillance, la cogestion garantit le maintien d'une concertation sociale permanente au sommet de l'entreprise. C'est sans doute cette cohésion sociale qui constitue l'un des éléments essentiels de la performance des entreprises allemandes. Outre la présentation des lois qui organisent la cogestion des salariés, la présente analyse se penche sur les différents régimes juridiques qui la composent et sur sa portée réelle au sein du conseil de surveillance.

MOTS CLÉS : Cogestion, participation des travailleurs, conseil de surveillance. 


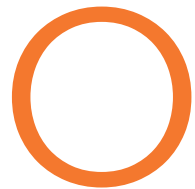

utre le système de négociation collective fondé sur l'autonomie contractuelle des organisations syndicales et patronales (Tarifautonomie), la cogestion des salariés (Mitbestimmung) constitue le second pilier sur lequel repose le partenariat social allemand ${ }^{1}$. Le système de cogestion repose sur une philosophie sociale spécifique fondée sur le principe d'équilibre et d'égalité (Gleichberechtigung) entre les droits du capital et ceux du travail. En effet, la cogestion répond à la logique selon laquelle le capital et le travail ont vocation à " cogérer » ou " co-déterminer » (mitbestimmen) la marche de l'entreprise. Elle entend compenser le poids considérable de l'employeur qui reste présent nonobstant l'existence des lois sociales et des conventions collectives ${ }^{2}$. Pour ce faire, elle instaure un partage du pouvoir entrepreneurial et se traduit par l'existence de régimes légaux de représentation permettant aux salariés, par l'entremise de représentants élus ou désignés, d'exercer un droit de contrôle et de participer à la gestion ainsi qu'à l'organisation de l'entreprise 3 .

Cependant, il convient de distinguer clairement la cogestion au niveau de l'établissement, c'est-à-dire, au sein de la structure réelle où travaillent les salariés, de la cogestion au sein du conseil de surveillance (Aufsichtsrat), qui est un organe de la société, c'est-à-dire de l'entité juridique propriétaire des actifs constituant l'entreprise. Au niveau de l'établissement, un comité d'établissement (Betriebsrat) doit être établi si l'établissement comporte un effectif d'au moins cinq salariés. La cogestion effectuée par le comité d'établissement permet une meilleure prise en compte des intérêts des salariés lorsqu'il s'agit de décisions qui ont un impact direct ou indirect sur leurs conditions de travail ${ }^{4}$. Ces décisions incluent, par exemple, l'instauration de nouvelles méthodes de travail, le remplacement de certains travailleurs, les accords sur l'extension ou la diminution des heures de travail, l'octroi de surévaluation tarifaire (übertarifliche Zulagen), la modification des heures de travail, ou la réduction des gratifications ${ }^{5}$.

1 R. Lasserre, "La cogestion allemande à l'épreuve de la globalisation », Regards sur l'économie allemande, $\mathrm{n}^{\circ} 72,2005$, p. 7 ; H. Otto, « Das konturlose Arbeitskampfrecht des BAG - Vom Arbeitskampf als ultima ratio zur nahezu unbeschränkten Kampffreiheit? », RdA, 2010, p. 135.

2 W. Hromadka et F. Maschmann, Arbeitsrecht Band 2, Kollektivarbeitsrecht und Arbeitsstreitigkeiten, 7ème éd., Heidelberg, Springer, 2017, p. 225.

3 H. Reichold, Betriebsverfassung als Sozialprivatrecht, Munich, Beck, 1995, p. 543; W. Hromadka et F. Maschmann, op. cit., $\mathrm{n}^{\circ} 2$, p. 225.

4 Voir aussi la contribution de Wolfgang Däubler dans ce numéro de la RDCTSS.

5 W. Hromadka et F. Maschmann, op. cit, n², p. 225. 
La cogestion au sein du conseil de surveillance n'est applicable que dans certains types de sociétés - principalement la société à responsabilité limitée ( $\mathrm{GmbH}$ ) ) et la société anonyme ( $A G$ ») - qui emploient plus de 500 salariés. Les représentants des salariés sont intégrés dans le conseil de surveillance qui, en principe, est un organe de la société où ne sont représentés que les associés. Il faut préciser, cependant, que le conseil de surveillance n'est pas l'organe de gestion. En droit des sociétés allemand, la gestion de la société est assurée par le directoire (Vorstand), le conseil de surveillance n'étant qu'un organe qui exerce un contrôle sur la gestion faite par le directoire. Par conséquent, les salariés membres du conseil de surveillance participent indirectement à la gestion de la société qu'assume le directoire. Cette forme de participation au sein du conseil de surveillance est dénommée " unternehmerische Mitbestimmung »(cogestion économique).

Il est important de souligner que le terme « Mitbestimmung » en droit allemand est habituellement traduit par le mot français « cogestion ». Les auteurs suivent cette tradition bien que le terme « cogestion » ne reflète pas correctement le rôle des salariés au sein des entreprises allemandes. En effet, il n'y a pas de participation des salariés au sein de l'organe de gestion (le directoire) ; les salariés participent plutôt uniquement au sein du conseil de surveillance qui est l'organe de contrôle de la sociétéb.

En droit allemand, plusieurs lois organisent la cogestion des salariés. Ces lois peuvent être classées selon qu'elles s'appliquent aux établissements ou aux sociétés ${ }^{7}$. Dans les établissements, la cogestion est régie par la Betriebsverfassungsgesetz du 25 septembre $2001^{8}$, la Europäische Betriebsrätegesetz du 28 octobre 1996', et la Sprecherausschussgesetz du 20 décembre $1988^{10}$. Quant à la cogestion des salariés dans les établissements des services publics, elle est organisée par la Bundespersonalvertretungsgesetz du 15 mars $1974^{11}$, ainsi que les Personalvertretungsgesetze des 16 Etats fédérés ${ }^{12}$.

Dans les sociétés, la cogestion est régie par la loi sur la cogestion des travailleurs (Mitbestimmungsgesetz) du 4 mai 197613, la Gesetz über die Drittelbeteiligung der Arbeitnehmer im Aufsichtsrat du 18 mai 200414, la Gesetz über die Mitbestimmung der Arbeitnehmer in den Aufsichtsräten und Vorständen der Unternehmen des Bergbaus und der Eisen und Stahl erzeugenden Industrie (Montan-Mitbestimmungsgesetz) du 21 mai $1951^{15}$, et la Gesetz zur Ergänzung des Gesetzes über die Mitbestimmung der Arbeitnehmer in den Aufsichtsräten und Vorständen der Unternehmen des Bergbaus und der Eisen und Stahl erzeugenden Industrie du 7 août $1956^{16}$.

6 C. Teichmann, "Le dirigeant d'Aktiengesellschaft en Allemagne ", in Y. Chaput et A. Lévi (dir.), La direction des sociétés anonymes en Europe, Paris, Litec, 2008, p. 13.

7 W. Hromadka et F. Maschmann, op. cit., n², p. 226.

8 Journal Officiel (BGBI. I) 2001, p. 2518.

9 Journal Officiel (BGBI. I) 1996, p. 1548.

10 BGBI. I, p. 2312.

11 BGBI I, p. 2312.

12 Voir W. Ilbertz, U. Widmaier et S. Sommer, Bundespersonalvertretungsgesetz mit Wahlordnung unter Einbeziehung der Landespersonalvertretungsgesetze, 13ème éd., Munich, Beck, 2015, para. 58 et s.

13 BGBI. I, p. 1153.

14 BGBI.I, p. 974.

15 BGBI.I, p. 347.

16 BGBII, p. 707. 
Pour les structures transnationales, il convient d'ajouter la Gesetz über die Beteiligung der Arbeitnehmer in der Europäischen Gesellschaft du 22 décembre 200417, la Gesetz über die Beteiligung der Arbeitnehmer in einer Europäischen Genossenschaft du 14 août $2006^{18}$, ainsi que la Gesetz über die Mitbestimmung der Arbeitnehmer bei einer grenzüberschreitenden Verschmelzung du 21 décembre $2006^{19}$.

Ce n'est que pour la cogestion dans les services publics qu'une seule des lois sur la cogestion des salariés est envisageable. Dans les autres cas, il est possible que plusieurs lois (jusqu'à quatre dans certaines situations) soient applicables concurremment. Par ailleurs, la « cogestion » au sein de l'établissement et la cogestion au sein du conseil de surveillance constituent des régimes différents qui peuvent, le cas échéant, s'appliquer concurremment dans une même entreprise ${ }^{20}$.

La présente étude a pour but de présenter les différents régimes juridiques qui la composent (I), ainsi que sa portée réelle au sein du conseil de surveillance (II).

\section{I - LES DEUX NIVEAUX DE LA COGESTION EN ALLEMAGNE}

Du point de vue de son champ d'application, la cogestion des salariés s'exerce à deux niveaux distincts: d'une part au niveau de l'établissement (Betrieb) (A) et d'autre part au niveau de l'entreprise (Unternehmen) (B) que la société organise juridiquement.

\section{A - LA COGESTION AU NIVEAU DE L'ÉTABLISSEMENT (BETRIEBLICHE MITBESTIMMUNG)}

Instaurée depuis 1952, la betriebliche Mitbestimmung a fait l'objet de plusieurs aménagements et constitue le régime de droit commun applicable à tous les établissements d'au moins cinq salariés. La betriebliche Mitbestimmung est un dispositif de concertation qui instaure un partage de pouvoir en matière de gestion suivant une répartition fine et équilibrée des prérogatives respectives de l'employeur et des représentants du personnel au sein du Betriebsrat (conseil d'établissement) ${ }^{21}$. D'une part, l'employeur conserve l'autonomie de décision économique ainsi que ses prérogatives en matière de contrat de travail. D'autre part, le Betriebsrat dispose d'un pouvoir d'information et de contrôle dans tous les domaines ou toutes les situations où les droits et intérêts sociaux des salariés peuvent être affectés par les décisions du chef d'entreprise. II convient de préciser que l'établissement n'a pas une capacité juridique en tant que tel. En effet, l'établissement constitue le lieu de travail des salariés; il peut être soit un site de production, soit une entité administrative de l'entreprise. Une entreprise peut maintenir plusieurs établissements, chacun d'entre eux ayant le droit de constituer son propre comité d'établissement.

17 BGBI I, p. 2675, 3686 pour la transposition de la Directive 2001/86/EG du 8 octobre 2001.

18 BGBI I, p. 1911, pour la transposition de la Directive 2003/72/EG du 8 octobre 2001.

19 BGBI I, p. 3332, pour la transposition de la Directive 2005/56/EG du 26 octobre 2005.

20 W. Hromadka et F. Maschmann, op. cit., n 2, p. 227.

21 Dans la cogestion au niveau de l'établissement, la Betriebsverfassung (constitution d'établissement) codifie la participation des salariés en déterminant les droits, obligations et pouvoirs respectifs du Betriebsrat (conseil d'établissement) qui est élu par le personnel. 
La cogestion au niveau de l'établissement régit les rapports sociaux au quotidien et reconnaît au Betriebsrat des compétences très larges englobant tous les aspects de la vie de l'établissement ${ }^{22}$. Ces compétences du Betriebsrat sont particulièrement importantes dans le domaine social. En effet, pour les questions relatives à la gestion collective du personnel et aux conditions de travail, les règles de cogestion ont pour conséquence que l'employeur ne peut valablement prendre de décision sans avoir préalablement consulté le Betriebsrat et obtenu son accord formel. Concernant les questions de personnel touchant à l'embauche, au licenciement ou toute autre question liée au contrat de travail individuel, le Betriebsrat dispose d'un droit de contrôle suspensif sur la régularité et l'opportunité sociale de la mesure. Pour ce qui est des questions économiques relatives à l'organisation de l'établissement ou de la production, le Betriebsrat a un pouvoir consultatif étendu lui permettant d'émettre des propositions laissées à la libre appréciation de l'employeur. Cependant, pour toute décision économique ayant des conséquences tangibles sur le personnel, l'employeur est tenu de présenter un plan social d'accompagnement dont les mesures compensatoires doivent obtenir l'accord du Betriebsrat.

\section{B - LA UNTERNEHMERISCHE MitBESTIMMUNG OU LA REPRÉSENTATION SALARIALE AU SEIN DU CONSEIL DE SURVEILLANCE}

Dans la unternehmerische Mitbestimmung, les salariés exercentleurs droits de cogestion par l'entremise de représentants élus ou désignés qui siègent dans les instances de contrôle de l'entreprise. Les représentants des salariés y disposent d'une voix délibérative aux côtés des représentants des propriétaires ou des actionnaires ${ }^{23}$. Cette forme de cogestion est réservée aux sociétés les plus importantes et fait l'objet de trois régimes distincts régis par des lois différentes: le regime instauré par la Montan-Mitbestimmungsgesetz de 1951 (1), celui prévu par la Drittelbeteiligungsgesetz de 2004 (2), et enfin celui organisé par la Mitbestimmungsgesetz de 1976 (3). Le champ d'application de chacune de ces lois est limité à des sociétés constituées sous une forme juridique déterminée et comprenant un seuil précis de salariés.

\section{La cogestion instaurée par la Montan-Mitbestimmungsgesetz de 1951}

La loi du 21 mai 1951 sur la cogestion dans les industries minières et sidérurgiques (Montan-Mitbestimmungsgesetz) constitue en droit allemand le tout premier texte adopté après la deuxième guerre mondiale en matière de cogestion des salariés ${ }^{24}$. Son champ d'application reste limité aux sociétés anonymes et aux sociétés à responsabilité limitée $(\mathrm{GmbH})$ du secteur minier et sidérurgique occupant plus de 1000 salariés ( $\S 1$ alinéa 2 Montan-Mitbestimmungsgesetz). Aux termes de cette loi, le conseil de surveillance est

22 II convient de noter cependant que, selon les domaines, ces attributions du Betriebsrat ont une portée très variable, allant d'un simple droit d'information à un véritable droit de codécision.

23 W. Hromadka et F. Maschmann, op. cit., n² 2, p. 226.

24 Sur I'histoire de la cogestion dans l'entreprise, voir T. Raiser, «Introduction », in T. Raiser, R. Veil et M. Jacobs (dir.), Mitbestimmungsgesetz und Drittelbeteiligungsgesetz, 6ème éd., Berlin, de Gruyter, 2015, Introduction, n 1-36; O. Sandrock et J. J. du Plessis, in J. J. du Plessis, B. Großfeld, C. Luttermann et al., German Corporate Governance in International and European Context,

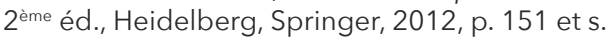


composé de onze membres : les travailleurs et les actionnaires sont représentés de manière paritaire par cinq membres, en raison de quatre membres plus un autre membre pour chaque groupe. Le $11^{\text {ème }}$ siège est attribué à une personnalité neutre. Il est impératif que deux des quatre représentants des salariés appartiennent à l'entreprise concernée et soient proposés par le conseil d'entreprise à l'organe chargé des élections. Quant aux deux autres membres et au cinquième représentant des salariés, ceux-ci sont également présentés par le comité d'entreprise, mais sur proposition des organisations centrales (Spitzenorganisationen) après une consultation préalable des syndicats représentés dans l'entreprise concernée. Aux termes des $\S \S 4,6$ et 8 de la Montan-Mitbestimmungsgesetz, la personne neutre susmentionnée est désignée par les membres du conseil de surveillance, puis proposée à l'organe en charge du vote (c'est-à-dire en principe l'assemblée des actionnaires). Outre la parité au sein du conseil de surveillance, la Montan-Mitbestimmungsgesetz accorde au personnel des entreprises auxquelles elle s'applique le droit d'être représenté au sein du directoire par un directeur du personnel (Arbeitsdirektor). La désignation et la révocation du directeur du personnel (Arbeitsdirektor) nécessite absolument l'aval de la majorité des travailleurs ( $\S 13$ Montan-Mitbestimmungsgesetz).

Il convient de noter qu'en plus de prévoir la forme la plus renforcée de la cogestion des salariés, la Montan-Mitbestimmungsgesetz occupe, du point de vue de la hiérarchie des normes, un rang supérieur aux autres textes en la matière ${ }^{25}$. Cette particularité se justifie par le fait qu'au moment de l'instauration de ce régime en 1952, les entreprises du secteur minier et sidérurgique avaient une importance capitale pour le développement économique de l'Allemagne. Partant, elles bénéficiaient d'une attention particulière. Aujourd'hui, le secteur minier et sidérurgique a presque disparu en Allemagne. Il en résulte que les sociétés auxquelles la Montan-Mitbestimmungsgesetz est applicable ne sont plus très nombreuses.

\section{La cogestion 'parité au tiers' instaurée par la Drittelbeteiligungsgesetz de 2004}

Le deuxième régime de cogestion est issu de la loi sur la constitution des entreprises (Betriebsverfassungsgesetz) du 19 juillet 1952, telle que modifiée par la

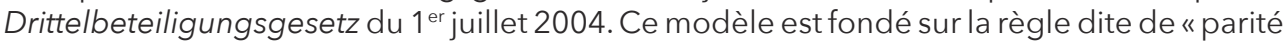
au tiers ». Son champ d'application est limité à la représentation des salariés au conseil de surveillance des sociétés auxquelles ne s'appliquent ni la Montan-Mitbestimmungsgesetz, ni la Mitbestimmungsgesetz de 1976. Ces sociétés sont la société anonyme (Aktiengesellschaft - AG), la société en commandite par actions (Kommanditgesellschaft auf Aktien - KGaA), la société à responsabilité limitée (Gesellschaft mit beschränkter Haftung - GmbH), la société d'assurance mutuelle (Versicherungsverein auf Gegenseitigkeit - WaG) et les coopératives économiques (Erwerbs- und Wirtschaftsgenossenschaften) ayant entre 501 et 2000 salariés (§ 1 alinéa 1 points 1-5 Drittelbeteiligungsgesetz).

Suivant ce modèle de cogestion, le conseil de surveillance est composé de 3 à 21 membres. Cependant, il est important de noter que le nombre de membres composant le conseil de surveillance doit être toujours impair. Si cette composition est telle que le nombre des représentants des salariés qui équivaut au tiers des membres est égal à trois ou plus, au moins deux d'entre eux doivent être choisis au sein de l'entreprise (c'est-à-dire

25 A. Seifert, "Actionnariat salarié et gouvernance d'entreprise en Allemagne », Bulletin de droit comparé du travail et de la sécurité sociale, 2001, p. 196. 
avoir la qualité de travailleurs), le reste pouvant provenir de «l'extérieur » (par exemple des délégués syndicaux). Au cas contraire, seuls les salariés de l'entreprise concernée peuvent être désignés pour représenter l'ensemble du personnel au consei ${ }^{26}$. Les représentants des salariés sont directement élus par leurs collègues.

\section{La cogestion « paritaire » instaurée par la Mitbestimmungsgesetz de 1976}

Le troisième et dernier modèle de cogestion est organisé par la loi du 4 mai 1976 (Mitbestimmungsgesetz). Ce modèle est considéré comme constituant la base légale la plus importante de la cogestion des salariés ${ }^{27}$. Son champ d'application couvre les sociétés organisées sous la forme de société anonyme, de société en commandite par actions, de société à responsabilité limitée ou de coopérative économique, pour autant qu'elles emploient plus de 2000 salariés (§ 1 alinéa 1 Mitbestimmungsgesetz). Cependant, la Mitbestimmungsgesetz régit les sociétés régies par le Montan-Mitbestimmungsgesetz de 1951, les communautés religieuses, les organisations de tendance (Tendenzunternehmen) politiques, caritatives, scientifiques ou culturelles, ainsi que les organisations religieuses à caractère philanthropique et éducatif dépendant de ces dernières (et ce, quelle que soit leur forme juridique $)^{28}$.

Le conseil de surveillance des sociétés auxquelles s'applique la Mitbestimmungsgesetz est composé respectivement de 12, 16 ou 20 membres selon que la société emploie moins de 10000 salariés, entre 10000 et 20000 salariés, ou plus de 20000 ( $\$ 7$ alinéa 1 phrase 1 points 1-3 Mitbestimmungsgesetz). Des dérogations peuvent être prévues par les statuts de chaque société. Cependant, ces dérogations ne sont admissibles que si elles revoient à la hausse les seuils mentionnés ci-haut (§ 7 alinéa 1 phrase 2 Mitbestimmungsgesetz). La répartition des sièges s'opère sur la base du principe paritaire selon lequel les salariés sont représentés à $50 \%$ d'une part et les actionnaires également à $50 \%$ d'autre part. Toutefois, cette parité est au désavantage des salariés en ce que le président du conseil de surveillance est régulièrement choisi parmi les représentants des actionnaires et sa voix est prépondérante en cas d'égalitée 29 .

Dans les conseils de surveillance ayant respectivement six ou huit représentants des salariés, quatre ou six d'entre eux doivent appartenir à la société concernée et avoir une ancienneté d'au moins une année. Quant aux deux autres, ils doivent être désignés par les syndicats qui, à leur tour, doivent également être représentés dans la société ou dans l'une de ses filiales (§ 7 alinéas 2 à 4, Mitbestimmungsgesetz). Pour les conseils de surveillance avec dix représentants des salariés, sept doivent être salariés et trois délégués syndicaux. Leur désignation est soumise aux mêmes conditions que celle des représentants évoqués ci-haut. Ils sont élus soit directement par les salariés, soit par leurs délégués selon que la société emploie moins de 8.000 travailleurs pour la première hypothèse ou plus pour la seconde hypothèse. Toutefois, ce principe est une solution subsidiaire ne valant que dans

$26 \S 4$ alinéa 2 Drittelbeteiligungsgesetz.

27 M. Henssler, "Arbeitnehmermitbestimmung im deutschen Gesellschaftsrecht », in T. Baums et P. Ulmer (dir.), Unternehmens-Mitbestimmung der Arbeitnehmer im Recht der EU-Mitgliedstaaten, Heidelberg, Recht und Wirtschaft GmbH, 2004, p. 133.

$28 \S 1^{\text {er }}$ alinéas 2 et 4 Mitbestimmungsgesetz.

29 Cette question est abordée en détail sous le point $D$ de la présente étude. Voir aussi R. Lasserre, art. préc. $\left(n^{\circ} 1\right)$, p. 9 ; A. Seifert, art. préc., $n^{\circ} 25$, p. 197. 
les cas où le personnel concerné par le vote n'aurait pas décidé autrement. Une telle décision nécessite une requête signée par un vingtième de salariés électeurs et ne peut passer que lorsque $50 \%$ de tous les salariés concernés ont pris part au vote et que la décision a recueilli la majorité des voix (§9 Mitbestimmungsgesetz). Le vote des représentants des salariés par les délégués choisis conformément aux paragraphes 12 et suivants de la Mitbestimmungsgesetz s'opère sur base des propositions émanant d'une part des salariés (§15 Mitbestimmungsgesetz) et d'autre part des syndicats ( 116 Mitbestimmungsgesetz).

Les différents régimes de la cogestion des salariés ici évoqués peuvent être résumés comme suit :

Aperçu des différents régimes de cogestion des salariés ${ }^{30}$

\begin{tabular}{|c|c|c|c|c|}
\hline $\begin{array}{l}\text { 1. Selon } \\
\text { l'entreprise } \\
\text { concernée }\end{array}$ & $\begin{array}{c}\text { Mitbestimmungs- } \\
\text { gesetz }\end{array}$ & $\begin{array}{c}\text { Montan- } \\
\text { mitbestimmungsgesetz } \\
(1951)\end{array}$ & $\begin{array}{l}\text { MitbestErG }{ }^{31} \\
(1956)\end{array}$ & $\begin{array}{l}\text { DrittelbG }{ }^{32} \\
(\mathbf{2 0 0 4 )}\end{array}$ \\
\hline $\begin{array}{l}\text { a) Forme } \\
\text { juridique }\end{array}$ & $\begin{array}{l}\text { Société anonyme, } \\
\text { société en } \\
\text { commandite simple } \\
\text { par actions, société } \\
\text { coopérative } \\
(\S 11 \mathrm{Nr} .1)\end{array}$ & $\begin{array}{l}\text { Société anonyme, société } \\
\text { à responsabilité limitée } \\
(\S 1 \mathrm{II})\end{array}$ & $\begin{array}{l}\text { Société anonyme, } \\
\text { société à responsabilité } \\
\text { limitée } \\
(\S 1)\end{array}$ & 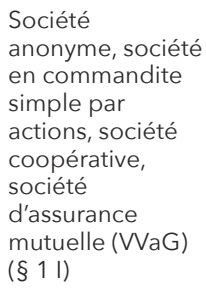 \\
\hline b) Taille & $\begin{array}{l}\text { Plus de } 2000 \text { salariés } \\
\text { (§ } 1 \text { I Nr. 2) }\end{array}$ & $\begin{array}{l}\text { Plus de } 1000 \text { salariés } \\
(\S 1 \mathrm{II})\end{array}$ & $\begin{array}{l}\text { Plus de } 1000 \text { salariés } \\
\text { au sein du groupe de } \\
\text { société } \\
(\S 1)\end{array}$ & $\begin{array}{l}\text { Plus de } 500 \\
\text { salariés } \\
(\S 1 \mathrm{I})\end{array}$ \\
\hline $\begin{array}{l}\text { c) Champ } \\
\text { d'application }\end{array}$ & $\begin{array}{l}\text { Ne vaut pas pour } \\
\text { les entreprises de } \\
\text { tendance (Tendenz- } \\
\text { unternehmen) } \\
\text { politique, caritatives, } \\
\text { scientifiques ou } \\
\text { culturelles, ainsi que } \\
\text { pour les organisations } \\
\text { religieuses } \\
(\S 1 \mathrm{IV})\end{array}$ & $\begin{array}{l}\text { Ne vaut que pour les } \\
\text { entreprises du secteur } \\
\text { minier } \\
(\S 1 \mid 1,2)\end{array}$ & $\begin{array}{l}\text { Ne vaut qu'au sein de } \\
\text { l'entreprise dominante } \\
\text { qui n'est pas sous le } \\
\text { régime de la } \\
\text { Montan- } \\
\text { mitbestimmungs-gesetz } \\
\text { (§3 I) }\end{array}$ & $\begin{array}{l}\text { Ne vaut que pour } \\
\text { les entreprises } \\
\text { de tendance } \\
\text { (Tendenz- } \\
\text { unternehmen) } \\
\text { politiques, } \\
\text { caritatives, } \\
\text { scientifiques ou } \\
\text { culturelles, ainsi } \\
\text { que pour les } \\
\text { organisations } \\
\text { religieuses et } \\
\text { leurs institutions } \\
\text { caritatives et } \\
\text { éducatives }\end{array}$ \\
\hline
\end{tabular}

30 Source: W. Hromadka et F. Maschmann, op. cit., n² 2, p. 239-241.

31 Gesetz zur Ergänzung des Gesetzes über die Mitbestimmung der Arbeitnehmer in den Aufsichtsräten und Vorständen der Unternehmen des Bergbaus und der Eisen und Stahl erzeugenden Industrie du 7 août 1956.

32 Gesetz über die Drittelbeteiligung der Arbeitnehmer im Aufsichtsrat du 18 mai 2004. 


\begin{tabular}{|c|c|c|c|c|}
\hline $\begin{array}{l}\text { 2. Selon le } \\
\text { conseil de } \\
\text { surveillance }\end{array}$ & $\begin{array}{c}\text { Mitbestimmungs- } \\
\text { gesetz }\end{array}$ & $\begin{array}{c}\text { Montan- } \\
\text { mitbestimmungsgesetz } \\
(1951)\end{array}$ & $\begin{array}{c}\text { MitbestErG } \\
(1956)\end{array}$ & $\begin{array}{l}\text { DrittelbG } \\
(2004)\end{array}$ \\
\hline a) Résumé & $\begin{array}{l}\text { Jusqu'à } 10000 \\
\text { salariés : } 6 \text { membres } \\
\text { pour les salariés et } \\
6 \text { membres pour les } \\
\text { actionnaires } \\
\\
\text { Jusqu'à } 20000 \\
\text { salariés : } 8 \text { membres } \\
\text { pour les salariés et } \\
8 \text { membres pour les } \\
\text { actionnaires } \\
\\
\text { Plus de } 20000 \\
\text { salariés: } \\
10 \text { membres pour les } \\
\text { salariés et } 10 \text { pour les } \\
\text { actionnaires } \\
\text { ( } ₹ 7 \text { I) } \\
\text { Parité }\end{array}$ & $\begin{array}{l}4 \text { représentants des } \\
\text { actionnaires et } 1 \text { autre } \\
\text { membre } \\
4 \text { représentants des } \\
\text { salariés et } 1 \text { autre } \\
\text { membre } \\
1 \text { autre membre } \\
\text { ( } \$ 4 \text { I) } \\
\text { Dans les sociétés avec } \\
\text { un capital nominal de } \\
\text { plus de : } \\
-10 \text { million d'Euros: } \\
\text { facultativement } 15 \\
\text { membres } \\
-25 \text { million d'Euros: } \\
\text { facultativement } 21 \\
\text { membres } \\
\text { ( } 9 \text { 9) } \\
\end{array}$ & $\begin{array}{l}7 \text { représentants des } \\
\text { actionnaires } \\
7 \text { représentants des } \\
\text { salariés } \\
1 \text { autre membre } \\
(\S 5 \mid 2) \\
\text { ( } \mid 2) \\
\text { Dans les sociétés de } \\
\text { capitaux de plus de } \\
25 \text { million d'euros: } \\
\text { facultativement } 21 \\
\text { membres ( } \$ 5 \mid 3)\end{array}$ & Parité à « $1 / 3$ » \\
\hline $\begin{array}{l}\text { b) } \\
\text { Représentant } \\
\text { des salariés }\end{array}$ & $\begin{array}{l}\text { Avec } 6 \text { membres : } \\
4 \text { salariés de } \\
\text { l'entreprise } \\
2 \text { représentants du } \\
\text { syndicat } \\
\\
\text { Avec } 8 \text { membres : } \\
6 \text { salariés de } \\
\text { l'entreprise } \\
2 \text { représentants du } \\
\text { syndicat } \\
\\
\\
\text { Avec } 10 \text { membres: } \\
7 \text { salariés de } \\
\text { l'entreprise } \\
3 \text { représentants du } \\
\text { syndicat } \\
\text { ( } \$ 7 \text { II) }\end{array}$ & $\begin{array}{l}2 \text { salariés de l'entreprise } \\
2 \text { membres et } 1 \text { autre } \\
\text { membre sur proposition } \\
\text { de l'organisation faîtière } \\
\text { (Spitzenorganisation) } \\
\text { (Exception : voir } \S 4 \text { II) } \\
\S \S 4 \text { । } 2 \text { lit. } B, 6 \text { । } 1\end{array}$ & $\begin{array}{l}5 \text { salariés de l'entreprise } \\
\text { appartenant au groupe } \\
\text { de sociétés } \\
2 \text { représentants des } \\
\text { syndicats } \\
(\S 6 \mid 2)\end{array}$ & $\begin{array}{l}\text { Au moins deux } \\
\text { salariés de } \\
\text { l'entreprise } \\
\text { (§ } 4 \text { II) }\end{array}$ \\
\hline
\end{tabular}




\section{II - PORTÉE DE LA COGESTION DES SALARIÉS AU SEIN DU CONSEIL DE SURVEILLANCE (AUFSICHTSRAT)}

Afin de saisir l'importance et la portée de la cogestion des salariés au sein de la société en droit allemand, il convient d'analyser les compétences du conseil de surveillance (A) et de déterminer qui, des salariés ou des actionnaires, ont prépondérance au sein du conseil de surveillance (B).

\section{A - LES COMPÉTENCES DU CONSEIL DE SURVEILLANCE}

Le droit allemand se singularise par une séparation stricte des fonctions de gestion et de celles de surveillance ${ }^{33}$. En effet, la gestion de la société anonyme revient au directoire (Vorstand), tandis que la surveillance du directoire est assurée par le conseil de surveillance (Aufsichtsrat). Contrairement au droit français, le droit allemand ne permet pas à une société de choisir entre un système moniste (avec un conseil d'administration) et un système dualiste (avec un directoire et un conseil de surveillance). En droit allemand, chaque société anonyme est obligée d'adopter le système dualiste et de former un directoire ainsi qu'un conseil de surveillance ${ }^{34}$. Cette séparation stricte des fonctions de gestion et de celles de surveillance s'opère par le biais de règles impératives qui imposent le respect des trois principes suivants :

- Une gestion indépendante assumée par le directoire ;

- La limitation du rôle du conseil de surveillance à sa seule fonction de contrôle ;

- L'impossibilité légale d'avoir simultanément un mandat au sein du directoire et du conseil de surveillance ${ }^{35}$.

Le directoire engage sa propre responsabilité concernant la gestion de la société ( $\$ 76$ Aktiengesetz $)^{36}$. Le conseil de surveillance ne peut exercer aucune fonction de gestion ${ }^{37}$. Cependant, il joue le rôle non seulement d'un organe de contrôle, mais aussi d'un organe de dialogue et de conseil. Dans les sociétés anonymes particulièrement, le conseil de surveillance constitue un organe de contrôle important en ce qu'il nomme et révoque les membres du directoire ( $\S 84$ alinéa $1^{\text {er }}$ Aktiengesetz), contrôle leurs actes ( $\$ 111$ alinéa 1 Aktiengesetz) et représente la société, le cas échéant, vis-à-vis des membres du directoire (§ 112 Aktiengesetz). II détermine aussi la rémunération des membres du directoire (§ 87 alinéa 1 Aktiengesetz) ${ }^{38}$. En outre, le conseil de surveillance peut conditionner la réalisation de certaines transactions à son accord préalable ( $\$ 111$ alinéa 4 Aktiengesetz). L'examen des comptes annuels consolidés de la société, qui peut avoir une influence sur la politique de distribution des dividendes, est une compétence conjointe du directoire et

33 C. Teichmann, art. préc., nº, p. 16.

34 La seule possibilité d'échapper à cette règle impérative consiste à transformer la société anonyme en société européenne qui a son propre système de participation des salariés.

$35 \S 105(1)$ Aktiengesetz: «Ein Aufsichtsratsmitglied kann nicht zugleich Vorstandsmitglied... sein ».

36 § 76 Aktiengesetz: «Der Vorstand hat unter eigener Verantwortung die Gesellschaft zu leiten ».

$37 \S 111(4) 1$ Aktiengesetz: «Maßnahmen der Geschäftsführung können dem Aufsichtsrat nicht übertragen werden». II convient de noter cependant que la doctrine demande de plus en plus souvent au conseil de surveillance d'assumer le rôle de conseiller du directoire.

38 Voir aussi § 107 alinéa 3 troisième phrase Aktiengesetz ainsi que la Gesetz zur Angemessenheit der Vortansvergütung du 5 août 2009 (BGBI. I, p. 2509). 
du conseil de surveillance ( $\S 172$ AktG en rapport avec $\S 264$ alinéa 1 deuxième phrase, Handelsgesetzbuch).

Au sein du conseil de surveillance des sociétés anonymes, les représentants des salariés siègent avec voix délibérative, à égalité de droits avec les représentants des actionnaires. Ils ont accès à toutes les informations relatives à la situation économique et financière de l'entreprise et sont associés à toutes les grandes décisions aussi bien en matière de répartition des bénéfices que de politique d'investissement. Ils participent aussi à toutes les décisions relatives au choix des dirigeants, aux opérations de fusion-acquisition et aux nouveaux projets de développement de la société. Ils ont donc un pouvoir de contrôle et de surveillance ${ }^{39}$. Ce pouvoir doit être exercé dans l'intérêt de toute l'entreprise et non pas dans le seul intérêt des salariés. En effet, il est vrai que les représentants des salariés sont naturellement plus proches des préoccupations des salariés. Néanmoins, ils commettraient une faute pouvant conduire à une obligation de payer des dommages et intérêts s'ils exerçaient leurs fonctions dans le seul intérêt des salariés.

Dans la société à responsabilité limitée $(\mathrm{GmbH})$, les associés désignent les gérants de la société (§ $46 \mathrm{Nr}$. $5 \mathrm{GmbHG}$ ). II en est de même des sociétés ayant une cogestion avec une parité à un tiers ${ }^{40}$. En revanche, dans les entreprises minières et dans les grandes entreprises, le conseil de surveillance a compétence pour désigner les gérants de la société comme c'est le cas dans les sociétés anonymes ( $\$ 12$ Montan-Mitbestimmungsgesetz, § 31 Mitbestimmungsgesetz). En outre, le conseil de surveillance décide de la rémunération des gérants ${ }^{41}$. Tout comme dans les sociétés anonymes, le conseil de surveillance des sociétés à responsabilité limitée du secteur minier peut faire dépendre de son accord préalable la prise de certaines décisions par la gérance ( $\$ 3$ Montan-Mitbestimmungsgesetz en rapport avec $\S 111$ alinéa 4 AktG). Cependant, l'assemblée générale peut imposer à la gérance de la société des instructions contraignantes, passant ainsi outre certaines décisions du conseil d'administration ${ }^{42}$.

39 W. Hromadka et F. Maschmann, op. cit., n 2, p. 235.

40 Comp. § 77 alinéa 1 deuxième phrase BetrVG 1952.

41 BGH 14 novembre 1983, Neue Juristische Wochenschrift (NJW) 1984, p. 733. II est important de noter que l'application des règles du directoire de la société anonyme au conseil de surveillance de la GmbH fait l'objet d'un débat. Pour plus de détails sur ce débat, voir V. Rieble et B. Schmittlein, Vergütung von Vorständen und Führungskräften Vergütungsregulierung durch VorstAG und Aufsichtsrecht, Munich, Beck, 2011, para. 32 et s. Néanmoins, l'application de ces règles au conseil de surveillance de la $\mathrm{GmbH}$ ne devrait pas être retenue du fait que ni la Mitbestimmungsgesetz, ni la $\mathrm{GmbHG}$ ne renvoient à la nouvelle formulation du § $87 \mathrm{AktG}$.

42 W. Zöllner, K.-G. Loritz et C. W. Hergenröder, Arbeitsrecht, 7’ème édition, Munich, Beck, 2015, § 53 III 3. 


\section{B - LA PRÉPONDÉRANCE DES ACTIONNAIRES AU SEIN DU CONSEIL DE SURVEILLANCE}

Nonobstant les règles de la cogestion des salariés, les actionnaires restent les acteurs prépondérants au sein du conseil de surveillance. Cette prépondérance des actionnaires est garantie non seulement en cas de participation paritaire des actionnaires et des salariés, mais aussi en cas de participation des salariés à un tiers des sièges du conseil de surveillance de la société.

En effet, il a déjà été noté que la Drittelbeteiligungsgesetz prévoit qu'un tiers des sièges du conseil de surveillance doit être occupé par des représentants des salariés. Le champ d'application de cette loi s'étend à toutes les sociétés anonymes et à toutes les sociétés à responsabilité limitée employant plus de 500 salariés. Dans un tel système de participation des salariés à hauteur d'un tiers du conseil de surveillance, les représentants des actionnaires ont une nette prépondérance car ils constituent les deux tiers du conseil. Partant, les représentants des actionnaires ont toujours la possibilité d'adopter des résolutions malgré l'opposition des représentants des salariés.

Cependant, la prépondérance des actionnaires semble moins évidente en cas de participation dite « paritaire ". Il a été relevé ci-avant qu'une telle participation est organisée par la Mitbestimmungsgesetz qui est applicable aux sociétés anonymes et aux sociétés à responsabilité limitée employant plus de 2000 salariés. Dans ces sociétés, les représentants des salariés constituent la moitié des membres du conseil de surveillance. L'on pourrait ainsi croire que les représentants des salariés ont le pouvoir de bloquer des décisions par leur vote, au détriment des actionnaires. Pourtant, tel n'est pas le cas car la prépondérance des actionnaires est assurée par l'existence de la deuxième voix décisive du président du conseil de surveillance. En effet, les règles de procédure d'élection du président du conseil de surveillance ont pour conséquence que le président du conseil de surveillance est pratiquement toujours un représentant des actionnaires. Plus particulièrement, le président du conseil de surveillance est élu au premier tour par tous les membres du conseil de surveillance. Si aucun candidat n'obtient la majorité, le président est élu au deuxième tour par les seuls représentants des actionnaires. Partant, le président est pratiquement toujours un représentant des actionnaires. Par conséquent, la participation dite « paritaire » n'est pas une représentation paritaire au sens strict ${ }^{43}$. II importe de souligner que ces dispositions qui garantissent la prépondérance de l'actionnariat constituent une condition essentielle permettant d'éviter que la cogestion ne constitue une violation du droit de propriété des actionnaires qui est garanti par la Constitution allemande (Grundgesetz) ${ }^{44}$.

43 C. Teichmann, art. préc., $n^{\circ}$ 6, p. 14.

44 Voirl'arrêtrenduparlaCourconstitutionnelle allemande(Bundesverfassungsgericht)sur la cogestion et publié dans le Recueil des arrêts de la Cour (Bundesverfassungsgerichtsentscheidungen, BVerfGE, 50, p. 290 et s.). 


\section{Conclusion et perspectives de réforme du régime de la cogestion des salariés}

Le régime de cogestion des salariés en droit allemand peut être considéré non seulement comme un facteur de cohésion sociale, mais aussi comme l'un des éléments essentiels de la performance des entreprises allemandes ${ }^{45}$. En effet, tout en ne portant pas atteinte à la prépondérance des actionnaires au sein du conseil de surveillance ${ }^{46}$, la cogestion garantit le maintien d'une concertation sociale permanente au sommet de l'entreprise. Elle permet aux salariés, grâce à leurs connaissances précises de la réalité de l'entreprise, de mieux faire valoir leurs intérêts et de rééquilibrer les débats au sein des instances décisionnelles ${ }^{47}$. La réussite du régime de la cogestion allemande est certainement due à l'esprit de compromis (Kompromissbereitschaft) qui caractérise la culture allemande. En effet, la recherche du consensus est perçue en Allemagne non pas comme une faiblesse, mais plutôt comme une force qui permet de conférer davantage de légitimité aux décisions ${ }^{48}$. C'est sans aucun doute cet aspect qui pourrait générer un enthousiasme pour le système allemand de la cogestion auprès des voisins de l'Allemagne, notamment la France.

Toutefois, il convient de relever que la cogestion telle qu'organisée depuis les années soixante-dix du dernier siècle ne fait pas I'unanimité en Allemagne. Il est vrai que la cogestion au sein du conseil de surveillance des grandes entreprises est régulièrement approuvée par presque tous les partis politiques allemands, en particulier par les chrétien-démocrates et les sociaux-démocrates. Même les représentants de l'industrie et du monde des affaires qui, après l'entrée en vigueur de la Mitbestimmungsgesetz en 1976, avaient dans un premier temps contesté la cogestion paritaire auprès de la Cour constitutionnelle ${ }^{49}$, ont finalement admis la présence des salariés au sein du conseil de surveillance. Cependant, il ne faut pas négliger le fait qu'un nombre considérable d'entreprises allemandes contournent les règles de la cogestion soit par la création d'une société européenne, soit par l'utilisation d'une société immatriculée à l'étranger sur base de la liberté d'établissement européenne ${ }^{50}$. II s'agit certes d'une minorité d'entreprises. Néanmoins, cette évolution confirme la nécessité d'une réforme des règles de la cogestion en droit allemand, d'autant plus que ces

45 R. Lasser, art. préc., $n^{\circ} 1$, p. 8.

46 M. Henssler, art. préc., $n^{\circ} 27$, p. 153-154.

47 R. Lasser, art. préc., $n^{\circ} 1$, p. 10.

48 J.-L. Beffa, La France doit choisir, Paris, Seuil, 2012, p. 210.

49 Voir ci-haut, note de bas de page $n^{\circ} 44$.

50 C. Teichmann, " Mitbestimmungserstreckung auf Auslandsgesellschaften », Zeitschrift für Wirtschaftsrecht (ZIP), 2016, p. 899. 


\section{LA COGESTION DES SALARIÉS EN DROIT ALLEMAND}

règles ne prennent pas en compte les travailleurs des entreprises allemandes employés à l'étranger ${ }^{51}$. Selon l'avis de plusieurs auteurs, une réforme de la cogestion allemande devrait prendre en compte le modèle européen consistant en une négociation entre les travailleurs et les organes de la société sur le système de cogestion ${ }^{52}$.

51 La Cour de Justice vient de confirmer que l'exclusion des salariés qui sont employés à l'étranger est conforme au droit de l'Union Européenne (C-566/15 (Erzberger/TUI AG), ECLI : EU : C : 2017 : 562).

52 Voir le résumé de la discussion par O. Sandrock et J. J. du Plessis, art. préc., n²4, p. 187-191. Pour une analyse plus récente, voir C. Teichmann, "Reform der Unternehmensmitbestimmung durch Einführung einer Verhandlungslösung », in M. Habersack, C. Behme, H. Eidenmüller et al. (dir.), Deutsche Mitbestimmung unter europäischem Reformzwang, Heidelberg, Deutscher Fachverlag $\mathrm{GmbH}, 2016$, p. 135-161.

\section{CHRISTOPHE TEICHMANN}

Professeur à l'Université de Würzburg (Julius-Maximilians-Universität Würzburg) .

Thèmes de recherche : Chaire de droit civil, droit commercial et droit des sociétés allemand et européen.

\section{Publications :}

C. Teichmann, "La rémunération des dirigeants dans un système dualiste d'administration ", dans Hayat/Reygrobellet (ed.), Le droit des affaires d'aujourd'hui à demain - Regards français et européens en hommage à Yves Chaput, LexisNexis, Paris, 2014, p. 321-344.

C. Teichmann, "Le dirigeant d'Aktiengesellschaft en Allemagne ", dans Chaput/Lévi (ed.), La direction des sociétés anonymes en Europe - Vers des pratiques harmonisées de gouvernance?, Litec, Paris, 2009, p. 9-54.

\section{JUSTIN MONSENEPWO}

Doctorant à la faculté de droit de I'Université de Würzburg (Julius-Maximilians-Universität Würzburg).

\section{Publications :}

Y. Manzanza Lumingu et J. Monsenepwo Mwakwaye (dir.), Droit, bonne gouvernance et développement durable. Mélanges en I'honneur du Professeur Jean-Michel Kumbu Ki Ngimbi, Paris, L'Harmattan, 2018

J. Monsenepwo, "Apport des instruments de la Conférence de La Haye au droit des affaires dans l'espace OHADA », Schriftenreihe Junges Afrikazentrum, n 5, 2016, p. 1-30. 